\title{
Retraction Note to: The hypoxia-inducible factor-1 regulates the microRNA185 expression through binding to hypoxia response elements sequence 2
}

\author{
Zhenguo Song $\cdot$ He Ren $\cdot$ Song Gao $\cdot$ Tiansuo Zhao $\cdot$ \\ Xiuchao Wang $\cdot$ Shengjie Zhang $\cdot$ Xiao Zhao $\cdot$ Lingling Jia \\ Junwei Sun · Jihui Hao
}

Published online: 9 January 2015

(c) Springer Science+Business Media New York 2014

Retraction Note to: Med Oncol (2013) 30:756

DOI: $10.1007 / \mathbf{s 1 2 0 3 2 - 0 1 3 - 0 7 5 6 - 8}$

This article was submitted to Medical Oncology without the knowledge of all authors and so the authors would like to retract the article. Dr. Song would like to extend an apology to the co-authors and readers for any inconvenience caused.

The online version of the original article can be found under doi:10.1007/s12032-013-0756-8.

Z. Song $\cdot$ H. Ren $\cdot$ S. Gao $\cdot$ T. Zhao $\cdot$ X. Wang $\cdot$ S. Zhang $\cdot$

X. Zhao $\cdot$ L. Jia $\cdot$ J. Sun $\cdot$ J. Hao $(\bowtie)$

Department of Pancreatic Cancer, Tianjin Medical University

Cancer Institute and Hospital, National Clinical Research Center

for Cancer, Key Laboratory of Cancer Prevention and Therapy,

Tianjin, China

e-mail: szzg1001@sina.com

Z. Song

Department of Anesthesiology and Operating Center, Tianjin

Medical University Cancer Institute and Hospital, National

Clinical Research Center for Cancer, Key Laboratory of Cancer

Prevention and Therapy, Tianjin, China 